\title{
Cosmological Special Theory of Relativity
}

\author{
Sangwha-Yi* \\ Department of Math, Taejon University 300-716, South Korea
}

*Corresponding Author: Sangwha-Yi, Department of Math, Taejon University 300-716, South Korea

Abstract: In the Cosmological Special Relativity Theory, we study Maxwell equations, electromagnetic wave equation and function.

Keywords: Special relativity theory; Electromagnetic field transformation; Electromagnetic wave equation; Maxwell equation

PACS Number: 03.30, 41.20

\section{INTRODUCTION}

Our article's aim is that we make cosmological special theory of relativity.

At first, Robertson-Walker metric is

$d \tau^{2}=d t^{2}-\frac{1}{c^{2}} \Omega^{2}(t)\left[\frac{d r^{2}}{1-k r^{2}}+r^{2} d \Omega^{2}\right]$

According to $\Lambda C D M$ model, our universe's $\mathrm{k}$ is zero. In this time, if $t_{0}$ is cosmological time[6],

$k=0, t=t_{0}>\Delta t, \Delta t$ is period of matter's motion

Hence, the proper time is in cosmological time,

$$
\begin{aligned}
& d \tau^{2}=d t^{2}-\frac{1}{c^{2}} \Omega^{2}\left(t_{0}\right)\left[d r^{2}+r^{2} d \Omega^{2}\right] \\
& =d t^{2}-\frac{1}{c^{2}} \Omega^{2}\left(t_{0}\right)\left[d x^{2}+d y^{2}+d z^{2}\right] \\
& =d t^{2}\left(1-\frac{1}{c^{2}} \Omega^{2}\left(t_{0}\right) V^{2}\right), \quad V^{2}=\frac{d x^{2}+d y^{2}+d z^{2}}{d t^{2}}
\end{aligned}
$$

In this time,

$$
d \bar{t}=d t, d \bar{x}=\Omega\left(t_{0}\right) d x, d \bar{y}=\Omega\left(t_{0}\right) d y, d \bar{z}=\Omega\left(t_{0}\right) d z
$$

Cosmological special theory of relativity's coordinate transformations are

$$
\begin{aligned}
& c \bar{t}=c t=\gamma\left(c \bar{t}+\frac{v_{0}}{c} \Omega\left(t_{0}\right) \bar{x}^{\prime}\right)=\gamma\left(c t+\frac{v_{0}}{c} \Omega\left(t_{0}\right) x ' \Omega\left(t_{0}\right)\right) \\
& \bar{x}=x \Omega\left(t_{0}\right)=\gamma\left(\bar{x}+v_{0} \Omega\left(t_{0}\right) \bar{t}^{\prime}\right)=\gamma\left(\Omega\left(t_{0}\right) x^{\prime}+v_{0} \Omega\left(t_{0}\right) t t^{\prime}\right)
\end{aligned}
$$


$\bar{y}=\Omega\left(t_{0}\right) y=\bar{y}^{\prime}=\Omega\left(t_{0}\right) y^{\prime}, \quad, \quad \gamma=1 / \sqrt{1-\frac{v_{0}^{2}}{c^{2}} \Omega^{2}\left(t_{0}\right)}$
$\bar{z}=\Omega\left(t_{0}\right) z=\bar{z}^{\prime}=\Omega\left(t_{0}\right) z^{\prime}$

Therefore, proper time is

$$
\begin{aligned}
& d \tau^{2}=d \bar{t}^{2}-\frac{1}{c^{2}}\left[d \bar{x}^{2}+d \bar{y}^{2}+d \bar{z}^{2}\right] \\
& =d t^{2}-\frac{1}{c^{2}} \Omega^{2}\left(t_{0}\right)\left[d x^{2}+d y^{2}+d z^{2}\right] \\
& =d t^{2}-\frac{1}{c^{2}} \Omega^{2}\left(t_{0}\right)\left[d x^{2}+d y^{R}+d z^{R}\right] \\
& =d \bar{t}^{2}-\frac{1}{c^{2}}\left[d \bar{x}^{2}+d \bar{y}^{2}+d \bar{z}^{R}\right]
\end{aligned}
$$

Hence, velocities are

$$
\begin{aligned}
& \frac{d x}{d t}=V_{x}=\frac{V_{x}+V_{0}}{1+\frac{\Omega^{2}\left(t_{0}\right)}{c^{2}} V_{x} !^{\prime} V_{0}}, V_{x}^{\prime}=\frac{d x^{\prime}}{d t^{\prime}} \\
& \frac{d y}{d t}=V_{y}=\frac{V_{y}^{\prime}}{\gamma\left(1+\frac{\Omega^{2}\left(t_{0}\right)}{c^{2}} V_{x} !^{\prime} V_{0}\right)}, V_{y}^{\prime}=\frac{d y^{\prime}}{d t^{\prime}} \\
& \frac{d z}{d t}=V_{z}=\frac{V_{z}^{\prime}}{\gamma\left(1+\frac{\Omega^{2}\left(t_{0}\right)}{c^{2}} V_{x} !^{\prime} V_{0}\right)}, V_{z}^{\prime}=\frac{d z^{\prime}}{d t^{\prime}}
\end{aligned}
$$

In cosmological special theory of relativity(CSTR)'s differential operators are

$$
\begin{aligned}
& \frac{1}{C} \frac{\partial}{\partial \bar{t}}=\frac{1}{C} \frac{\partial}{\partial t}=\gamma\left(\underset{C}{\frac{1}{\partial \bar{t}^{\prime}}} \frac{\partial}{C}-\frac{V_{0}}{C} \Omega\left(t_{0}\right) \frac{\partial}{\partial \bar{x}^{\prime}}\right) \\
& =\gamma\left(\frac{1}{C} \frac{\partial}{\partial t^{\prime}}-\frac{V_{0}}{C} \frac{\partial}{\partial x^{\prime}}\right) \\
& \frac{\partial}{\partial \bar{x}}=\frac{\partial}{\partial x} \frac{1}{\Omega\left(t_{0}\right)}=\gamma\left(\frac{\partial}{\partial \bar{x}^{\prime}}-\frac{V_{0}}{C} \Omega\left(t_{0}\right) \frac{1}{C} \frac{\partial}{\partial \bar{t}^{\prime}}\right) \\
& =\gamma\left(\frac{\partial}{\partial x} \frac{1}{\Omega\left(t_{0}\right)}-\frac{V_{0}}{C} \Omega\left(t_{0}\right) \frac{1}{C} \frac{\partial}{\partial t^{\prime}}\right) \\
& \frac{\partial}{\partial \bar{y}}=\frac{\partial}{\partial y} \frac{1}{\Omega\left(t_{0}\right)}=\frac{\partial}{\partial \bar{y}^{\prime}}=\frac{\partial}{\partial y}, \frac{1}{\Omega\left(t_{0}\right)} \\
& \frac{\partial}{\partial \bar{z}}=\frac{\partial}{\partial z} \frac{1}{\Omega\left(t_{0}\right)}=\frac{\partial}{\partial \bar{z}^{\prime}}=\frac{\partial}{\partial z}, \frac{1}{\Omega\left(t_{0}\right)}, \gamma=1 / \sqrt{1-\frac{V_{0}^{2}}{C^{2}} \Omega^{2}\left(t_{0}\right)}
\end{aligned}
$$

Hence,

$$
\frac{1}{c^{2}} \frac{\partial^{2}}{\partial \bar{t}^{2}}-\bar{\nabla}^{2}=\frac{1}{c^{2}} \frac{\partial^{2}}{\partial t^{2}}-\frac{1}{\Omega^{2}\left(t_{0}\right)}\left\{\left(\frac{\partial}{\partial x}\right)^{2}+\left(\frac{\partial}{\partial y}\right)^{2}+\left(\frac{\partial}{\partial z}\right)^{2}\right\}
$$


$=\frac{1}{c^{2}} \frac{\partial^{2}}{\partial t^{2}}-\frac{1}{\Omega^{2}\left(t_{0}\right)}\left\{\left(\frac{\partial}{\partial x^{\prime}}\right)^{2}+\left(\frac{\partial}{\partial y^{\prime}}\right)^{2}+\left(\frac{\partial}{\partial z^{\prime}}\right)^{2}\right\}$

The electric charge density $\rho$ and the electric current density $\vec{j}$ are

$$
j^{\mu}=\rho_{0} \frac{d x^{\mu}}{d \tau}, j^{0}=c \rho=c \gamma \rho_{0}, \dot{j}^{i}=\vec{j}=\rho \vec{u}, \quad i=1,2,3
$$

In CSTR, transformations of the electric charge density and the electric current density are likely as coordinate transformations are

$$
\begin{aligned}
& c \bar{\rho}=c \rho=\gamma\left(c \bar{\rho}+\frac{V_{0}}{c} \Omega\left(t_{0}\right) \bar{j}_{x}^{\prime}\right)=\gamma\left(c \rho+\frac{v_{0}}{c} \Omega\left(t_{0}\right) j_{x} ' \Omega\left(t_{0}\right)\right) \\
& \left.\bar{j}_{x}=j_{x} \Omega\left(t_{0}\right)=\gamma\left(\bar{j}_{x}+v_{0} \Omega\left(t_{0}\right) \bar{\rho}^{\prime}\right)=\gamma \Omega\left(t_{0}\right) j_{x}+v_{0} \Omega\left(t_{0}\right) \rho{ }^{\prime}\right) \\
& \bar{j}_{y}=\Omega\left(t_{0}\right) j_{y}=\bar{j}_{y}^{\prime}=\Omega\left(t_{0}\right) j_{y}^{\prime} ', \quad, \gamma=1 / \sqrt{1-\frac{v_{0}^{2}}{c^{2}} \Omega^{2}\left(t_{0}\right)} \\
& \bar{j}_{z}=\Omega\left(t_{0}\right) j_{z}=\bar{j}_{z}^{\prime}=\Omega\left(t_{0}\right) j_{z}^{\prime}, \quad \gamma
\end{aligned}
$$

\section{ELECTROdynAMiCS IN CSTR}

The electromagnetic potential $A^{\mu}$ is 4-vector potential.Hence, transformations of $A^{\mu}$ are

$$
\begin{aligned}
& \left.\bar{\phi}=\phi=\gamma \bar{\phi}+\frac{V_{0}}{C} \Omega\left(t_{0}\right) \bar{A}_{x}^{\prime}\right)=\gamma\left(\phi^{\prime}+\frac{V_{0}}{C} \Omega\left(t_{0}\right) A_{x} ' \Omega\left(t_{0}\right)\right) \\
& \bar{A}_{x}=A_{x} \Omega\left(t_{0}\right)=\gamma\left(\bar{A}_{x}+\frac{V_{0}}{C} \Omega\left(t_{0}\right) \bar{\phi}^{\prime}\right)=\gamma\left(\Omega\left(t_{0}\right) A_{x}+\frac{V_{0}}{C} \Omega\left(t_{0}\right) \phi^{\prime}\right) \\
& \bar{A}_{y}=\Omega\left(t_{0}\right) A_{y}=\bar{A}_{y}^{\prime}=\Omega\left(t_{0}\right) A_{y}^{\prime}{ }^{\prime}, \quad, \gamma=1 / \sqrt{1-\frac{V_{0}^{2}}{C^{2}} \Omega^{2}\left(t_{0}\right)} \\
& \bar{A}_{z}=\Omega\left(t_{0}\right) A_{z}=\bar{A}_{z}^{\prime}=\Omega\left(t_{0}\right) A_{z}^{\prime}, \quad \gamma
\end{aligned}
$$

In CSTR, electric field $\stackrel{\vec{E}}{\overrightarrow{ }}$ and magnetic field $\stackrel{\vec{B}}{\vec{b}}$ have to satisfy Maxwell equations of special relativity theory. Hence, in CSRT, Maxwell equations are likely as special theory of relativity,

$$
\begin{aligned}
& \overrightarrow{\vec{\nabla}} \cdot \overrightarrow{\vec{E}}=4 \pi \bar{\rho} \\
& \overrightarrow{\vec{\nabla}} \cdot \overrightarrow{\vec{B}}=0 \\
& \overrightarrow{\vec{\nabla}} \times \overrightarrow{\vec{E}}=-\frac{1}{C} \frac{\partial \overrightarrow{\vec{B}}}{\partial \bar{t}} \\
& \overrightarrow{\vec{\nabla}} \times \overrightarrow{\vec{B}}=\frac{1}{C} \frac{\partial \vec{E}}{\partial \bar{t}}+\frac{4 \pi}{C} \vec{j}
\end{aligned}
$$

In this time, $\mathrm{Eq}(13-\mathrm{i})$ is

$$
\vec{\nabla} \cdot \overrightarrow{\vec{E}}=\frac{1}{\Omega\left(t_{0}\right)} \vec{\nabla} \cdot \overrightarrow{\vec{E}}=4 \pi \bar{\rho}=4 \pi \rho
$$

Hence, $\vec{E}=\vec{E} \Omega\left(t_{0}\right)$.According to special relativity, $\vec{B}=\vec{B} \Omega\left(t_{0}\right)$ 
$\mathrm{Eq}(13-\mathrm{ii})$ is

$$
\overrightarrow{\vec{\nabla}} \cdot \overrightarrow{\vec{B}}=\frac{1}{\Omega\left(t_{0}\right)} \vec{\nabla} \cdot \vec{B} \Omega\left(t_{0}\right)=\vec{\nabla} \cdot \vec{B}=0
$$

$\mathrm{Eq}(13-\mathrm{iii})$ is

$$
\overrightarrow{\vec{\nabla}} \times \overrightarrow{\vec{E}}=\frac{1}{\Omega\left(t_{0}\right)} \vec{\nabla} \times \vec{E} \Omega\left(t_{0}\right)=\vec{\nabla} \times \vec{E}=-\frac{1}{c} \frac{\partial \vec{B}}{\partial \bar{t}}=-\frac{1}{c} \frac{\partial \vec{B}}{\partial t} \Omega\left(t_{0}\right)
$$

$\mathrm{Eq}(13-\mathrm{iv})$ is

$$
\begin{aligned}
& \overrightarrow{\vec{\nabla}} \times \overrightarrow{\vec{B}}=\frac{1}{\Omega\left(t_{0}\right)} \vec{\nabla} \times \vec{B} \Omega\left(t_{0}\right)=\vec{\nabla} \times \vec{B} \\
& =\frac{1}{C} \frac{\partial \vec{E}}{\partial \bar{t}}+\frac{4 \pi}{C} \vec{j}=\Omega\left(t_{0}\right)\left(\frac{1}{C} \frac{\partial \vec{E}}{\partial t}+\frac{4 \pi}{C} \vec{j}\right)
\end{aligned}
$$

Hence, in CSTR, Maxwell equations are

$$
\begin{aligned}
& \vec{\nabla} \cdot \vec{E}=4 \pi \rho \\
& \vec{\nabla} \cdot \vec{B}=0 \\
& \vec{\nabla} \times \vec{E}=-\frac{1}{C} \frac{\partial \vec{B}}{\partial t} \Omega\left(t_{0}\right) \\
& \vec{\nabla} \times \vec{B}=\Omega\left(t_{0}\right) \underset{C}{\left(\frac{1}{2} \frac{\partial \vec{E}}{\partial t}+\frac{4 \pi}{C} \vec{j}\right)}
\end{aligned}
$$

Therefore, in CSTR, the electric field $\vec{E}$ and the magnetic field $\vec{B}$ are

$$
\begin{aligned}
& \overrightarrow{\vec{E}}=\vec{E} \Omega\left(t_{0}\right)=\Omega\left(t_{0}\right)\left(-\vec{\nabla} \phi-\frac{1}{C} \frac{\partial \vec{A}}{\partial t}\right) \\
& =-\Omega\left(t_{0}\right) \vec{\nabla} \phi-\Omega\left(t_{0}\right) \frac{1}{C} \frac{\partial \vec{A}}{\partial t}=-\overrightarrow{\bar{\nabla}}\left(\bar{\phi} \Omega^{2}\left(t_{0}\right)\right)-\frac{1}{C} \frac{\partial \overrightarrow{\vec{A}}}{\partial \bar{t}} \\
& \overrightarrow{\vec{B}}=\vec{B} \Omega\left(t_{0}\right)=\Omega\left(t_{0}\right) \vec{\nabla} \times \vec{A}=\Omega\left(t_{0}\right) \vec{\nabla} \times \overrightarrow{\vec{A}}
\end{aligned}
$$

\section{Electromagnetic Wave in CSTR}

Electromagnetic wave equation is in CSTR,

$$
\begin{aligned}
& \left.\frac{1}{c} \frac{\partial}{\partial t} \vec{\nabla} \times \vec{E}\right)=-\Omega\left(t_{0}\right) \frac{1}{c^{2}} \frac{\partial^{2} \vec{B}}{\partial t^{2}} \\
& =\vec{\nabla} \times\left(\frac{1}{C} \frac{\partial \vec{E}}{\partial t}\right)=\vec{\nabla} \times\left(\frac{1}{\Omega\left(t_{0}\right)} \vec{\nabla} \times \vec{B}\right), \vec{\nabla} \times \vec{j}=\overrightarrow{0} \\
& \left.=\frac{1}{\Omega\left(t_{0}\right)}\left\{-\nabla^{2} \vec{B}+\vec{\nabla} \vec{\nabla} \cdot \vec{B}\right)\right\}=-\frac{1}{\Omega\left(t_{0}\right)} \nabla^{2} \vec{B}
\end{aligned}
$$

Hence, electromagnetic wave equation is

$$
\Omega\left(t_{0}\right) \frac{1}{c^{2}} \frac{\partial^{2} \vec{B}}{\partial t^{2}}-\frac{1}{\Omega\left(t_{0}\right)} \nabla^{2} \vec{B}=\overrightarrow{0}
$$


And,

$$
\begin{aligned}
& \left.\frac{1}{C} \frac{\partial}{\partial t} \vec{\nabla} \times \vec{B}\right)=\Omega\left(t_{0}\right) \frac{1}{C^{2}} \frac{\partial^{2} \vec{E}}{\partial t^{2}}, \frac{1}{C} \frac{\partial \vec{j}}{\partial t}=\overrightarrow{0} \\
& =\vec{\nabla} \times\left(\frac{1}{C} \frac{\partial \vec{B}}{\partial t}\right)=\vec{\nabla} \times\left(-\frac{1}{\Omega\left(t_{0}\right)} \vec{\nabla} \times \vec{E}\right) \\
& \left.=-\frac{1}{\Omega\left(t_{0}\right)}\left\{-\nabla^{2} \vec{E}+\vec{\nabla} \vec{\nabla} \cdot \vec{E}\right)\right\}=\frac{1}{\Omega\left(t_{0}\right)} \nabla^{2} \vec{E}, \vec{\nabla}(4 \pi \rho)=\overrightarrow{0}
\end{aligned}
$$

Hence, electromagnetic wave equation is

$\Omega\left(t_{0}\right) \frac{1}{C^{2}} \frac{\partial^{2} \vec{E}}{\partial t^{2}}-\frac{1}{\Omega\left(t_{0}\right)} \nabla^{2} \vec{E}=\overrightarrow{0}$

In CSTR, electromagnetic wave functions are

$$
\begin{aligned}
& \vec{E}=\vec{E}_{0} \sin \Phi, \vec{B}=\vec{B}_{0} \sin \Phi \\
& \Phi=\omega\left\{\frac{t}{\sqrt{\Omega\left(t_{0}\right)}}-\frac{\sqrt{\Omega\left(t_{0}\right)}}{c}\left(x_{x}+m y+n z\right)\right\}
\end{aligned}
$$

Where,

$$
I^{2}+m^{2}+n^{2}=1
$$

According to Maxwell equations are in CSTR,[1]

$$
\begin{array}{ll}
\Omega\left(t_{0}\right)\left\{\frac{1}{C} \frac{\partial E_{x}}{\partial t}+\frac{4 \pi}{C} j_{x}\right\}=\left(\frac{\partial B_{z}}{\partial y}-\frac{\partial B_{y}}{\partial z}\right), & \Omega\left(t_{0}\right) \frac{1}{C} \frac{\partial B_{x}}{\partial t}=\left(\frac{\partial E_{y}}{\partial z}-\frac{\partial E_{z}}{\partial y}\right) \\
\Omega\left(t_{0}\right)\left\{\frac{1}{C} \frac{\partial E_{y}}{\partial t}+\frac{4 \pi}{C} j_{y}\right\}=\left(\frac{\partial B_{x}}{\partial z}-\frac{\partial B_{z}}{\partial x}\right), & \Omega\left(t_{0}\right) \frac{1}{C} \frac{\partial B_{y}}{\partial t}=\left(\frac{\partial E_{z}}{\partial x}-\frac{\partial E_{x}}{\partial z}\right) \\
\Omega\left(t_{0}\right)\left\{\frac{1}{C} \frac{\partial E_{z}}{\partial t}+\frac{4 \pi}{C} j_{z}\right)=\left(\frac{\partial B_{y}}{\partial x}-\frac{\partial B_{x}}{\partial y}\right), & \Omega\left(t_{0}\right) \frac{1}{C} \frac{\partial B_{z}}{\partial t}=\left(\frac{\partial E_{x}}{\partial y}-\frac{\partial E_{y}}{\partial x}\right)
\end{array}
$$

Where,

$$
\begin{aligned}
& \Omega\left(t_{0}\right)\left\{\frac{1}{C} \frac{\partial E_{x}^{\prime}}{\partial t}+\frac{4 \pi}{C} j_{x}\right\}=\left\{\frac{\partial}{\partial y} \gamma\left(B_{z}+\frac{V_{0}}{C} \Omega\left(t_{0}\right) E_{y}^{\prime}\right)-\frac{\partial}{\partial z} \gamma\left(B_{y}+\frac{V_{0}}{C} \Omega\left(t_{0}\right) E_{z}^{\prime}{ }^{\prime}\right)\right\} \\
& \Omega\left(t_{0}\right)\left\{\frac{1}{C} \frac{\partial}{\partial t} \gamma\left(E_{y}+\frac{V_{0}}{C} \Omega\left(t_{0}\right) B_{z}^{\prime}\right)+\frac{4 \pi}{C} j_{y}\right\}=\left\{\frac{\partial B_{x}^{\prime}}{\partial z}-\frac{\partial}{\partial x} \gamma\left(B_{z}+\frac{V_{0}}{C} \Omega\left(t_{0}\right) E_{y}^{\prime}{ }^{\prime}\right)\right\} \\
& \Omega\left(t_{0}\right)\left\{\frac{1}{C} \frac{\partial}{\partial t} \gamma\left(E_{z}+\frac{V_{0}}{C} \Omega\left(t_{0}\right) B_{y}^{\prime}\right)+\frac{4 \pi}{C} j_{z}\right\}=\left\{\frac{\partial}{\partial y} \gamma\left(B_{y}+\frac{V_{0}}{C} \Omega\left(t_{0}\right) E_{z}^{\prime}\right)-\frac{\partial B_{x}^{\prime}}{\partial z}\right\}
\end{aligned}
$$

Where,[1]

$$
\begin{aligned}
& \Omega\left(t_{0}\right) \frac{1}{C} \frac{\partial B_{x}^{\prime}}{\partial t}=\left\{\frac{\partial}{\partial z} \gamma\left(E_{y}+\frac{V_{0}}{C} \Omega\left(t_{0}\right) B_{z}^{\prime}\right)-\frac{\partial}{\partial y} \gamma\left(E_{z}-\frac{V_{0}}{C} \Omega\left(t_{0}\right) B_{y}^{\prime}{ }^{\prime}\right)\right\} \\
& \Omega\left(t_{0}\right) \frac{1}{C} \frac{\partial}{\partial t} \gamma\left(B_{y}+\frac{V_{0}}{C} \Omega\left(t_{0}\right) E_{z}^{\prime}\right)=\left\{\frac{\partial}{\partial x} \gamma\left(E_{z}-\frac{V_{0}}{C} \Omega\left(t_{0}\right) B_{y}^{\prime}\right)-\frac{\partial E_{x}^{\prime}}{\partial z}\right\} \\
& \Omega\left(t_{0}\right) \frac{1}{C} \frac{\partial}{\partial t} \gamma\left(B_{z}+\frac{V_{0}}{C} \Omega\left(t_{0}\right) E_{y}^{\prime}\right)=\left\{\frac{\partial E_{x}^{\prime}}{\partial y}-\frac{\partial}{\partial x} \gamma\left(E_{y}+\frac{V_{0}}{C} \Omega\left(t_{0}\right) B_{z}^{\prime}\right\}\right.
\end{aligned}
$$


Hence, in CSTR, transformations of electromagnetic field are

$$
\begin{aligned}
& E_{x}=E_{x}{ }^{\prime}, E_{y}=\gamma\left(E_{y}{ }^{\prime}+\frac{v_{0}}{c} \Omega\left(t_{0}\right) B_{z}{ }^{\prime}\right), E_{z}=\gamma\left(E_{z}{ }^{\prime}-\frac{v_{0}}{c} \Omega\left(t_{0}\right) B_{y}{ }^{\prime}\right) \\
& B_{x}=B_{x}{ }^{\prime}, B_{y}=\gamma\left(B_{y}{ }^{\prime}-\frac{v_{0}}{c} \Omega\left(t_{0}\right) E_{z}{ }^{\prime}\right), B_{z}=\gamma\left(B_{z}{ }^{\prime}+\frac{v_{0}}{c} \Omega\left(t_{0}\right) E_{y}{ }^{\prime}\right)
\end{aligned}
$$

In CSTR, electromagnetic wave functions are

$$
\begin{aligned}
& E_{x}{ }^{\prime}=E_{x 0} \sin \Phi^{\prime}, E_{y}{ }^{\prime}=\gamma\left(E_{y 0}-\frac{v_{0}}{c} \Omega\left(t_{0}\right) B_{z 0}\right) \sin \Phi^{\prime}, E_{z}{ }^{\prime}=\gamma\left(E_{z 0}+\frac{v_{0}}{c} \Omega\left(t_{0}\right) B_{y 0}\right) \sin \Phi^{\prime} \\
& B_{x}{ }^{\prime}=B_{x 0} \sin \Phi^{\prime}, B_{y}{ }^{\prime}=\gamma\left(B_{y 0}+\frac{v_{0}}{c} \Omega\left(t_{0}\right) E_{z 0}\right) \sin \Phi^{\prime}, B_{z}{ }^{\prime}=\gamma\left(B_{z 0}-\frac{v_{0}}{c} \Omega\left(t_{0}\right) E_{y 0}\right) \sin \Phi^{\prime}
\end{aligned}
$$

In this time,

$$
\begin{aligned}
& \Phi^{\prime}=\omega^{\prime}\left\{\frac{t^{\prime}}{\sqrt{\Omega\left(t_{0}\right)}}-\frac{\sqrt{\Omega\left(t_{0}\right)}}{c}\left(I^{\prime} x^{\prime}+m^{\prime} y^{\prime}+n^{\prime} z^{\prime}\right)\right\} \\
& \Phi=\omega\left\{\frac{t}{\sqrt{\Omega\left(t_{0}\right)}}-\frac{\sqrt{\Omega\left(t_{0}\right)}}{c}(x+m y+n z)\right\}
\end{aligned}
$$

If we compare $\mathrm{Eq}(34)$ and $\mathrm{Eq}(35)$,

$$
\omega^{\prime}=\omega \gamma\left(1-l \frac{v_{0}}{c}\right), l^{\prime}=\frac{l-\frac{v_{0}}{c}}{1-l \frac{v_{0}}{c}}, m^{\prime}=\frac{m}{\gamma\left(1-l \frac{v_{0}}{c}\right)}, n^{\prime}=\frac{n}{\gamma\left(1-l \frac{v_{0}}{c}\right)}
$$

Where,

$$
I^{R}+m^{R}+n^{R}=1
$$

\section{CONClusion}

We know Maxwell equations, electromagnetic wave equations and functions in Cosmological Special Theory of Relativity.

\section{REFERENCES}

[1]A. Einstein, “ Zur Elektrodynamik bewegter K"orper”, Annalen der Physik. 17:891(1905)

[2]Friedman-Lemaitre-Robertson-Walker metric-Wikipedia

[3]A.Miller, Albert Einstein's Special Theory of Relativity(Addison-Wesley Publishing Co., Inc., 1981)

[4]W.Rindler, Special Relativity(2nd ed., Oliver and Boyd, Edinburg,1966)

[5]D.J. Griffith,” Introduction To Electrodynamics”,(2nd ed.,Prentice Hall,Inc.1981)

[6]Lambda-CDM model -Wikipedia

Citation: Sangwha-Yi, (2020). Cosmological Special Theory of Relativity. International Journal of Advanced Research in Physical Science (IJARPS) 7(11), pp.4-9, 2020.

Copyright: (๑ 2020 Authors, This is an open-access article distributed under the terms of the Creative Commons Attribution License, which permits unrestricted use, distribution, and reproduction in any medium, provided the original author and source are credited. 